\title{
Controlled loop and graft formations of water-soluble polymers on SAMs for the design of biomaterials surfaces
}

\author{
Ko Yamada ${ }^{1}$, Ryo Katoono ${ }^{1,2}$ and Nobuhiko Yui ${ }^{1,2}$
}

We prepared four types of water-soluble polymers with anchoring group(s) at one or both terminals: poly(ethylene glycol) (PEG) with one or two anchoring groups and polyrotaxane with one or two anchoring groups. Each polymer was immobilized to successfully form a loop or graft structure on a gold substrate covered with a self-assembled monolayer (SAM) of a tri(ethylene glycol)-dodecanethiol conjugate. Controlled immobilization of the polymers onto a gold substrate was examined by an original two-step protocol and was confirmed by quartz crystal microbalance (QCM) measurement. We investigated the effects of the immobilization modes (loop and graft) of the polymers on the surface properties of biomaterials. Surface softness or rigidity of the hydrated polymers on a SAM was analyzed by QCM with dissipation monitoring measurements, by which we found smaller ratios of energy dissipation to frequency $(\Delta D / \Delta f)$ for a loop structure than for a graft structure in an aqueous environment. With regard to surface wettability, substrates with PEG or polyrotaxane were similar in terms of static and dynamic contact angles and were also not influenced by the immobilization modes. We demonstrate that less fibrinogen was adsorbed on the polyrotaxane loop, making it a good platform for the design of biomaterials surfaces.

Polymer Journal (2012) 44, 286-293; doi:10.1038/pj.2011.130; published online 21 December 2011

Keywords: loop and graft formations; low protein adsorption; poly(ethylene glycol) (PEG); polyrotaxane; quartz crystal microbalance with dissipation monitoring (QCM-D); Self-Assembled Monolayer (SAM)

\section{INTRODUCTION}

Preventing nonspecific interactions in biological systems is a major concern in the design of biomaterials surfaces. ${ }^{1}$ Much effort has been devoted to the development of polymers to eliminate protein adsorption and the subsequent cellular responses on surfaces contacting blood or body fluid in a living body., ${ }^{2,3}$ In particular, hydrophilic polymers such as poly(ethylene glycol) $(\mathrm{PEG})^{4-8}$ and phospholipid polymers $^{9-13}$ have been well developed and are some of the most promising candidates to eliminate protein adsorption. They have a suitable chain length and density, excluded volume effect, entropic repulsive force, surface wettability and hydration. However, a mechanistic understanding is still incomplete because these factors are interdependent. Other studies have reported that a surface with some ethylene glycol units forming a self-assembled monolayer (SAM) on gold can effectively reduce protein adsorption, reasoning that the surface was completely covered with well-hydrated ethylene glycol units, which decreased protein adsorption. ${ }^{14-16}$ In this context, the net effect of polymeric architectures on the surface properties of biomaterials should be further examined.

In addition to the above numerous efforts, we are particularly interested in the state of polymers immobilized on a surface, and envisage two types of immobilization modes (loop and graft), ${ }^{17-20}$ as shown in Figure 1. On a surface, one or both terminals of a linear polymeric chain are immobilized though an anchoring group to form a graft or loop structure, and the rest of the surface is covered with a SAM of a conjugate containing an oligo(ethylene glycol). Any difference in the macromolecular conformation of a water-soluble polymer would be provided by immobilization under the different modes and would affect the surface properties. We envision two further characteristics (soft and rigid) for each mode by tuning the polymer (Figure 1). Thus, we designed PEG chains $\mathbf{1} \boldsymbol{l}$ and $\mathbf{1} \boldsymbol{g}$ (soft) attached to a thiol group as an anchoring group at both and one terminal, respectively, and water-soluble polyrotaxanes $2 l$ and $2 g$ (rigid) in which the same PEG chain exists and is threading into the cavity of a partially methylated $\alpha$-cyclodextrin ( $\alpha$-CD; Figure 2 ). The alignment of cyclic molecules along a chain would affect the macromolecular conformation to modulate flexibility in such a supramolecular architecture. We immobilized these four water-soluble polymers through $\mathrm{Au}-\mathrm{S}$ bond $(\mathrm{s})^{21}$ on a gold substrate covered with a SAM of a tri(ethylene glycol)-dodecanethiol (TEGDT) conjugate 3 (Figure 2). Attention must also be paid to the methodology of controlled immobilization, which is described in detail later as a two-step protocol. Here, we report the controlled loop and graft formations of water-soluble polymers $\mathbf{1}$ and $\mathbf{2}$ on gold through the quartz crystal

${ }^{1}$ School of Materials Science, Japan Advanced Institute of Science and Technology, Ishikawa, Japan and ${ }^{2}$ JST, CREST, Tokyo, Japan

Correspondence: Current address: Professor N Yui, Institute of Biomaterials and Bioengineering, Tokyo Medical and Dental University, 2-6-10 Kanda-Surugadai, Chiyoda-ku, Tokyo 101-0062, Japan.

E-mail: yui.org@tmd.ac.jp

Received 28 August 2011; revised and accepted 18 October 2011; published online 21 December 2011 


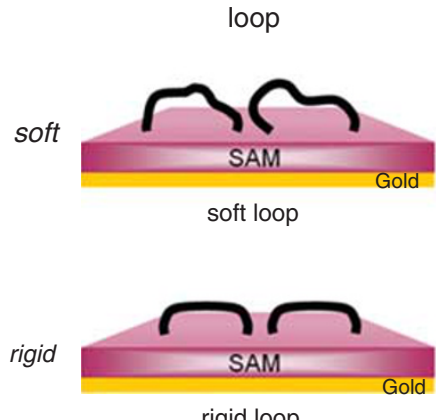

rigid loop
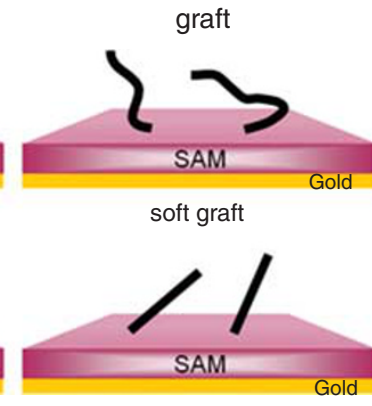

rigid graft

Figure 1 Illustration of polymer-immobilized surfaces with four different states on self-assembled monolayer (SAM).

microbalance (QCM) technique. We also report a preliminary investigation of the surface properties in terms of surface softness in an aqueous environment by QCM with dissipation monitoring (QCMD), static and dynamic contact angles and protein adsorption using surface plasmon resonance (SPR) spectroscopy.

\section{EXPERIMENTAL PROCEDURE}

\section{Materials}

PEG (average molecular weight 3000, CAS No. 25322-68-3) was purchased from Wako Pure Chemicals Industries, Ltd. (Osaka, Japan) and used as a starting material for preparing PEG bis(2-aminoethyl) ether (PEGBA $\mathbf{4}^{18}$ MALDI-TOF MS $m / z 2987\left([\mathrm{M}(n=65)+\mathrm{Na}]^{+}, 100 \%\right)$. A QCM gold (111) electrode was purchased from Q-sense Inc. (Gothenburg, Sweden). A gold (111) substrate for contact angle measurements was kindly provided by the Ishihara group (The University of Tokyo, Japan). Silicon wafers were purchased from Furuuchi Chemical Co. (Tokyo, Japan); the surface of the silicon wafers was covered with $\sim 10$-nm-thick $\mathrm{SiO}_{2}$ layers. A gold-evaporated substrate was prepared on the silicon wafer by plasma sputtering with a 3.0-nm-thick adhesion layer of $\mathrm{Cr}$, followed by $27 \mathrm{~nm}$ of $\mathrm{Au}$ (SCOTT-C3, Ulvac Kiko Inc., Kanagawa, Japan). The $\mathrm{O}_{2}$-plasma treatment was performed with an $\mathrm{O}_{2}$-plasma generator (PR500, Yamato Scientific Co., Ltd., Tokyo, Japan) before the plasma sputtering of $\mathrm{Cr}$ and $\mathrm{Au}$. A gold sensor chip for SPR measurements was purchased from GE Healthcare Co. (Stockholm, Sweden). For the investigation of protein adsorption, human serum albumin (HSA, lyophilized powder, 96-99\%, A1653) and human plasma fibrinogen (HPF, 55-75\% protein, $\geqslant 65 \%$ of protein is clottable, F4129) were purchased from Sigma Co. (St Louis, MO, USA). A phosphate-buffered saline (PBS) solution (10 M, pH 7.4) was purchased from Invitrogen Co. (Carlsbad, CA, USA) and used after dilution $(1 \mathrm{M})$. Synthetic procedures and spectral data of new compounds $(\mathbf{1}-\mathbf{3}$, $\mathbf{5}, \mathbf{6}, \mathbf{8}, \mathbf{1 1 - 1 3}$ ) are described in the Supplementary Information (Supplementary Scheme S1-S3). Abbreviations for some reagents (Supplementary Scheme 1-2) are as follows: DMT-MM (4-(4,6-dimethoxy-1,3,5-triazin-2-yl)-4-methylmorpholinium chloride), BOP (benzotriazole-1-yloxytris(dimethylamino)phosphonium hexafluorophosphate), HOBt ( $N$-hydroxybenzotriazole) and DIEA ( $N, N$-diisopropylethylamine).

Preparation of PEG and polyrotaxane loops (IL and IIL) and PEG and polyrotaxane grafts (IG and IIG) on SAM (two-step protocol)

Step one: solutions of PEG 1/polyrotaxane 2 with concentrations $\left(10^{-16}\right.$ $10^{-15} \mathrm{M}$ for $1 / 10^{-7}-10^{-6} \mathrm{M}$ for 2 ) in methanol/water containing $0.1 \mathrm{~N} / 1 \mathrm{~N} \mathrm{NaOH}$ were prepared by stirring for $30 \mathrm{~min}$ to produce a thiol group at the terminal(s) of $\mathbf{1 / 2}$. Three gold electrodes were immersed in each of the above basic solutions for $10-60 \mathrm{~s} / 5-60 \mathrm{~min}$ to prepare substrates $\mathbf{I}^{1 \mathrm{st}} \mathbf{a}-\mathbf{c} / \mathbf{I I}^{1 \mathrm{st}} \mathbf{a}-\mathbf{c}$. They were then washed with water to remove physically adsorbed $\mathbf{1 / 2}$ and dried in vacuo before recording QCM measurements.

Step two: the substrates $\mathbf{I}^{1 \mathrm{st}} \mathbf{a}-\mathbf{c} / \mathbf{I I}^{1 \mathrm{st}} \mathbf{a}-\mathbf{c}$ were immersed in solutions of $\mathbf{3}$ $\left([3]=1.0 \times 10^{-3} \mathrm{M}\right)$ in $50 \%$ ethanol/water containing $0.1 \mathrm{~N} \mathrm{NaOH}$ for $6 \mathrm{~h}$, washed with ethanol and water and subsequently dried in vacuo to prepare substrates $\mathbf{I}^{\text {2nd }} \mathbf{a}-\mathbf{c} / \mathbf{I I}^{2 \text { nd }} \mathbf{a}-\mathbf{c}$.

The simplest reference III was prepared by immersion of an electrode in a solution of $3\left([3]=1.0 \times 10^{-3} \mathrm{M}\right)$ in $50 \%$ ethanol/water containing $0.1 \mathrm{~N} \mathrm{NaOH}$ for $6 \mathrm{~h}$, followed by washing with ethanol and water and drying in vacuo.

\section{Confirmation of loop formation}

Each of the substrates $\mathbf{I}^{\text {stt }} \mathbf{L a}-\mathbf{c} / \mathbf{I}{ }^{15 t} \mathbf{L a}-\mathbf{c}$ and $\mathbf{I}^{2 n d} \mathbf{L a}-\mathbf{c} / \mathbf{I I}^{\text {2nd }} \mathbf{L a}$-c was immersed in an aqueous solution of colloidal silvers ${ }^{18,21,22}\left(1 \times 10^{-5} \mathrm{M}\right)$ for a period of $4 \mathrm{~h}$, washed with water and subsequently dried in vacuo to measure a change in frequency $(\Delta f)$ using QCM (see $\mathbf{I}^{1 \mathrm{st}} \boldsymbol{L}, \mathbf{I}^{\text {2nd }} \boldsymbol{L}, \mathbf{I I}^{1 \mathrm{st}} \boldsymbol{L}$, and $\mathbf{I I}^{2 \mathrm{end}} \boldsymbol{L}$ in Figure 3 ).

The substrates $\mathbf{I}^{1 \mathrm{st}} \mathbf{L a} \mathbf{a}-\mathbf{c} / \mathbf{I I}^{1 \text { st }} \mathbf{L a} \mathbf{a}-\mathbf{c}$ were also simply incubated by immersion in a blank solution ( $50 \%$ ethanol/water containing $0.1 \mathrm{~N} \mathrm{NaOH}$ but not containing 3 ) for $6 \mathrm{~h}$ to yield control substrates, denoted as $\mathbf{I}^{\text {inc }} \boldsymbol{L a - c}$ or ${ }^{\text {inc }} \boldsymbol{L a} \mathbf{L}-\mathbf{c}$. Further, these control substrates were immersed in a colloidal silver solution $\left(1 \times 10^{-5} \mathrm{M}\right)$ in the same manner as for $\mathbf{I}^{\mathbf{1 s t}} \mathbf{L} \mathbf{a}-\mathbf{c} / \mathbf{I I}{ }^{\text {st }} \boldsymbol{L} \mathbf{a}-\mathbf{c}$ and $\mathbf{I}^{\text {2nd }} \boldsymbol{L a}-\mathbf{c} / \mathbf{I I}^{2 \text { nd }} \mathbf{L a}-\mathbf{c}$ to measure $\Delta f$.

\section{Measurements}

QCM/QCM-D was measured with Q-sense E1 (Q-Sense Inc.) in an ambient/ aqueous atmosphere. All the QCM/QCM-D measurements were recorded at a controlled temperature of $25 \pm 0.1{ }^{\circ} \mathrm{C}$. The analysis was carried out using the frequency change obtained from a frequency of $45 \mathrm{~Hz}$. Mass increases on a gold substrate at the first step for immobilization and the second step for SAM formation were monitored from changes in the frequency $(\Delta f)$ using the Sauerbrey equation. ${ }^{23,24}$ The energy dissipation change $(\Delta D)$ during the twostep protocol was calculated by turning off the electric field and monitoring the decay process of the oscillation after immersion of the samples in water for 5 days.

Contact angle measurement. Static and dynamic contact angle measurements were carried out using a contact angle goniometer (DM-501, Kyowa Interface Science Co. Ltd., Saitama, Japan) equipped with a CCD camera, a motordriven microsyringe and a processing computer. Before recording the measurements, the surface tension of water $\left(71.8 \pm 1 \mathrm{mN} \mathrm{m}^{-1}\right)$ was confirmed using the pendant drop method with a Young-Laplace curve-fitting algorithm in the instrument's software (FAMAS software, Kyowa Interface Science Co. Ltd., Saitama, Japan). For static contact angle measurement, droplets of either water or methylene iodide were delivered to five different locations on a sample surface using the microsyringe set by the computer for a flow rate of $2.0 \mu \mathrm{min}^{-1}$ for $4.7 \mathrm{~s}$ at $2{ }^{\circ} \mathrm{C}$ under a relative humidity of $45 \%$. The dispersion/polar component of the surface tension of each sample was calculated from the static contact angles of water and methylene iodide using the equation reported by Owens and Wendt. ${ }^{25}$ For dynamic contact angle measurement, water drops were delivered to five different locations on a sample surface in the same manner as described above, but at a different flow rate $\left(2.0 \mu \mathrm{l} \mathrm{min}^{-1}\right.$ for $8.0 \mathrm{~s}$, or $8.0 \mathrm{ml} \mathrm{min}^{-1}$ for $2.0 \mathrm{~s}$ ), and then drawn from the surface at the same rate as delivered. In both measurements, the droplet images on surfaces were captured by the camera. Their profiles were then fitted using the FAMAS software to determine the contact angles. All the samples were measured three times after immersion in water for 5 days.

Plasma protein adsorption. Adsorption of albumin and fibrinogen onto sample surfaces were measured with an SPR sensor apparatus (Biacore 1000, GE Healthcare Co.). Two kinds of proteins, HSA $\left(4.5 \mathrm{mg} \mathrm{ml}^{-1}\right)$ and HPF $\left(0.3 \mathrm{mg} \mathrm{ml}^{-1}\right)$, in a $1 \mathrm{M}$ PBS solution $(\mathrm{pH} 7.4)$ were passed over the sample surface of the sensor chip set up in the instrument through a flow cell at a flow rate of $15 \mu \mathrm{min}^{-1}$ for $5 \mathrm{~min}$ or $1 \mu \mathrm{min}^{-1}$ for $75 \mathrm{~min}$ at $37^{\circ} \mathrm{C}$. The sample surfaces were rinsed with the PBS for $5 \mathrm{~min}$. A surface protein coverage of $0.1 \mathrm{ng} \mathrm{cm}^{-2}$ was assumed from the resonance unit in the SPR sensorgram in this study. The measurements were repeated three times after immersion in PBS for 5 days.

\section{RESULTS AND DISCUSSION}

Preparation of PEG $(1 \mathrm{l} / \mathrm{g})$ and polyrotaxane $(2 \mathrm{l} / \mathrm{g})$ for immobilization in loop or graft mode on SAM

The PEG chain $\mathbf{l} l$ for loop formation was prepared by a condensation reaction of PEGBA 4 (average $n=65$ ) at each terminal with a tyrosine derivative $\mathbf{5}$ tethering an anchoring group. The PEG chain $\mathbf{1} \boldsymbol{g}$ for 


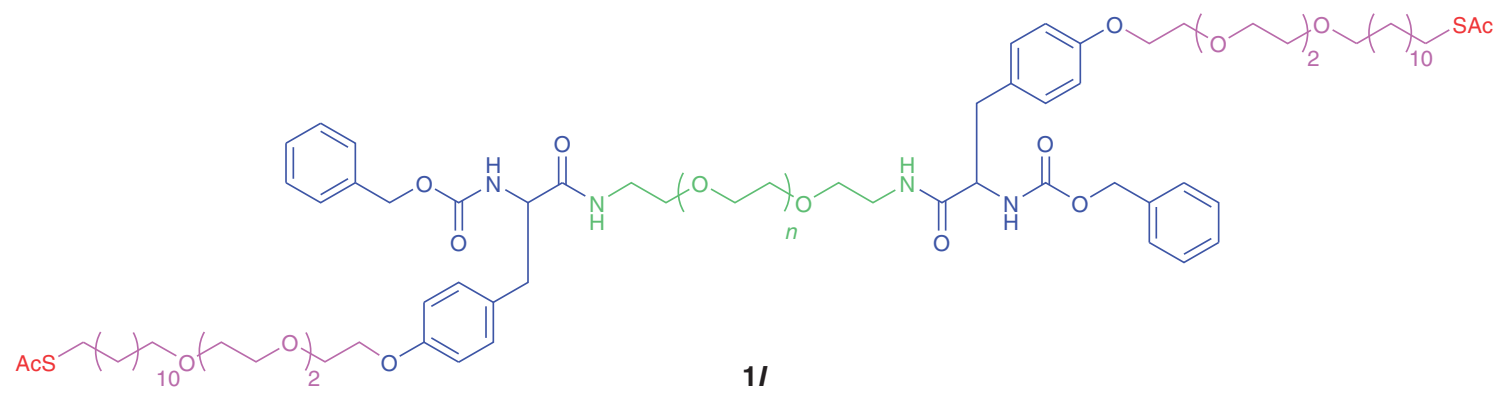

$1 /$

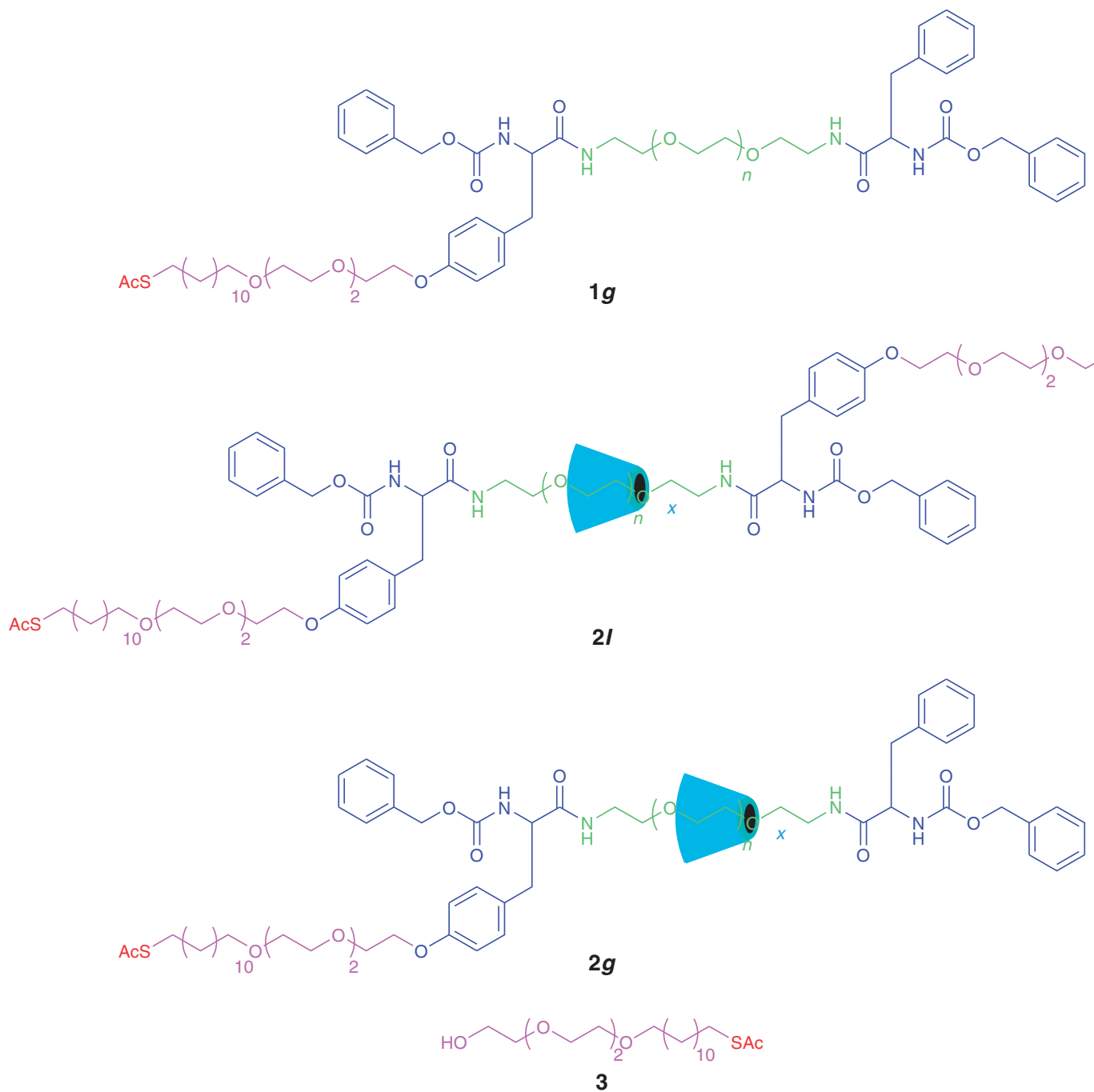

Figure 2 Chemical structures of poly(ethylene glycol)s $\mathbf{1}$ and $\mathbf{1} \boldsymbol{g}$ and polyrotaxanes $\mathbf{2} \boldsymbol{I}$ and $\mathbf{2} \boldsymbol{g}$ with anchoring group(s) for immobilization, and tri(ethylene glycol)-dodecanethiol $\mathbf{3}$ for self-assembled monolayer formation.

graft formation was obtained by similar reactions with $\mathbf{5}$ and a phenylalanine analog 7, which is not attached to any anchoring group, in a stepwise manner (Figure 2 and Scheme 1). The water-soluble polyrotaxane $\mathbf{2 l / g}$ for loop or graft formation was prepared by the endcapping of a pseudopolyrotaxane $4 \cdot \alpha-C^{18}$ / $6 \cdot \alpha-C D$, which was obtained by mixing $4 / 6$ with $\alpha-C D$ in water, with tyrosine $5 /$ phenylalanine 7 , followed by partial methylation of $\alpha$-CD in polyrotaxane $8 \mathrm{l} / \mathrm{g}$ to attain water solubility (Figure 2 and Scheme 2). Structures of the polyrotaxanes 2 were characterized by ${ }^{1} \mathrm{H}$ nuclear magnetic resonance spectra of $\mathbf{8 l} / \mathbf{g}$ and $2 \boldsymbol{l} / \mathbf{g}$ measured in $\mathrm{D}_{2} \mathrm{O}$ containing $1 \mathrm{wt} \% \mathrm{NaOD}$ and $\mathrm{D}_{2} \mathrm{O}$, showing that the ratio $(x)$ of $\alpha$-CD to PEG was $19 / 26$ for $\mathbf{8 l} / \mathbf{g}$ and the number $(m)$ of methyl groups per $\alpha$-CD in $2 l / g$ was $11 / 11$ on average (Supplementary Figure S1 in Supplementary Information). Gel permeation chromatography profiles of $\mathbf{2} \boldsymbol{l}$ and $\mathbf{2} \boldsymbol{g}$ are depicted in Supplementary Figure S2 in the Supplementary Information. The TEGDT conjugate $\mathbf{3}$ for SAM formation was obtained by coupling tert-butyl\{2-[2-(2chloroethoxy)ethoxy] ethoxy\}dimethylsilane ${ }^{26}$ and $S$-acetyl-protected dodecanethiol $^{27}$ through Williamson etherification, followed by desilylation. The TEGDT conjugate 3 was also used to prepare the tyrosine derivative $\mathbf{5}$. The preparation of $\mathbf{3}$ and $\mathbf{5}$ is described in detail in the Supplementary Information (Supplementary Scheme S1). 


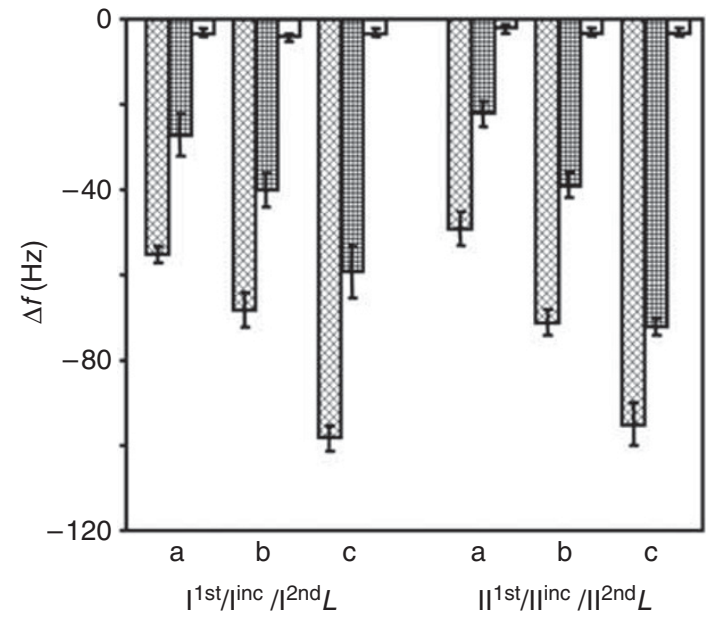

Figure 3 Changes in frequency $(\Delta f)$ upon conjugation of colloidal silver $\left(1 \times 10^{-5} \mathrm{M}\right)$ to a terminal thiol group of poly(ethylene glycol) $1 /$ and

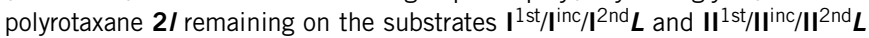

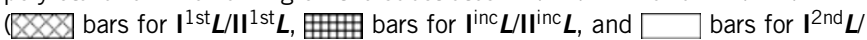
$\left.\|{ }^{2 n d} \boldsymbol{L}\right)$, measured by quartz crystal microbalance $(n=3)$.

\section{Preparation of PEG (1l/g)- and polyrotaxane $(2 \mathrm{l} / \mathrm{g})$-immobilized} substrates $I^{2 n d} L / G$ and $I^{2 n d} L / G$ in loop or graft mode on SAM The loop formation of PEG $\mathbf{l} l$ and polyrotaxane $2 \boldsymbol{l}$ on SAM was achieved by the following the two-step protocol, as illustrated in Scheme 3. In the first step, the acetyl group at each terminal of $1 / / 2 l$ was hydrolyzed in methanol/water containing $0.1 \mathrm{~N} / 1 \mathrm{~N} \mathrm{NaOH}$ to produce an intermediary thiol group. A gold electrode was then immersed for $10-60 \mathrm{~s}$ and $5-60 \mathrm{~min}$ to yield substrates $\mathbf{I}^{1 \mathrm{st}} \boldsymbol{L}$ and $\mathbf{I I}^{1 \text { st }} \boldsymbol{L}$, respectively. Further, in the second step, the substrates $\mathbf{I}^{1 \text { st }} \boldsymbol{L}$ and $\mathbf{I I}^{1 \mathrm{st}} \boldsymbol{L}$ were immersed in a solution of TEGDT conjugate $\mathbf{3}$ in $50 \%$ EtOH/water containing $0.1 \mathrm{~N} \mathrm{NaOH}$ for $6 \mathrm{~h}$ to yield substrates $\mathbf{I}^{2 \mathrm{nd}} \boldsymbol{L}$ and $\mathbf{I I}^{2 \text { nd }} \boldsymbol{L}$, respectively. At each step, loop formation was confirmed, as described later in detail (vide infra). The PEG $\mathbf{1} \boldsymbol{g}$ - and polyrotaxane $\mathbf{2 g}$-grafted substrates $\mathbf{I}^{2 \text { nd }} \boldsymbol{G}$ and $\mathbf{I I}^{2 \text { nd }} \boldsymbol{G}$ were prepared by a similar twostep protocol, using $\mathbf{l} \boldsymbol{g}$ and $\mathbf{2} \boldsymbol{g}$ instead of $\mathbf{1} \boldsymbol{l}$ and $\boldsymbol{2} \boldsymbol{l}$. The TEGDT conjugate 3 was immobilized to yield substrate III as the simplest control. Detailed conditions concerning solution concentration and immersion time upon immobilization of $\mathbf{1 - 3}$ are summarized in Table 1. These immobilization processes were monitored by QCM measurement after drying the substrates.

The amounts of $\mathbf{1 l} / \mathbf{g}$ and $\mathbf{2 l} / \mathbf{g}$ immobilized onto a gold electrode in the first step were defined as the number of molecules per $100 \mathrm{~nm}^{2}$. The amounts were successfully controlled to be 1,2 and $3\left(/ 100 \mathrm{~nm}^{2}\right)$ (a, b, and c) by solution concentrations of $1 l / g$ and $2 l / g$ and immersion time (Table 1). It is noteworthy that almost the same increase $\left(\Delta m_{2-1}: 1.8 \times 10 \mathrm{ng} \mathrm{cm}^{-2}\right)$ was observed in the mass of the substrates for all substrates $\mathbf{I}^{\text {2nd }}$ and $\mathbf{I I}^{2 \text { nd }}$ upon the immobilization of 3 in the second step, corresponding to an increase $\left(1.8 \times 10 \mathrm{ng} \mathrm{cm}^{-2}\right)$ in mass of the control substrate III. This result suggests that the TEGDT conjugate $\mathbf{3}$ was successfully self-assembled onto all substrates in the second step. The numbers of $\mathrm{OH}$ and $\mathrm{OMe}$ groups on substrates $\mathbf{I}^{2 \text { nd }}, \mathbf{I I}^{\text {2nd }}$ and III are also summarized in Table 1 for ease of reference. The obtained chain density was not very high in each substrate compared with those $\left(0.1-0.7 \mathrm{~nm}^{-2}\right)$ for PEG grafts $(\mathrm{MW}=750$, 2000 or 5000) reported in previous studies for effective minimization of protein adsorption. ${ }^{7}$

Loop formation requires the formation of an $\mathrm{Au}-\mathrm{S}$ bond at each terminal of a chain. The increase in mass of a substrate indicates the amount of a chain immobilized on the substrate in QCM measurement, as described above (Table 1). However, it does not indicate how the chain is immobilized, whether as loop or graft. To qualitatively investigate the immobilization modes of PEG $\mathbf{1} \boldsymbol{l}$ and polyrotaxane $\mathbf{2} \boldsymbol{l}$ on the substrates $\mathbf{I}^{1 \mathrm{st}} \boldsymbol{L} / \mathbf{I}^{2 \mathrm{nd}} \boldsymbol{L}$ and $\mathbf{I I}^{1 \mathrm{st}} \boldsymbol{L} / \mathbf{I I}^{2 \mathrm{nd}} \boldsymbol{L}$ (Scheme 3), QCM measurement was carried out again on the conjugation of colloidal silver (see Confirmation of loop formation, Experimental Procedure). A frequency change $(\Delta f)$ would be monitored on the conjugation through Ag-S bond formation only when $1 / / 2 l$ is immobilized in graft mode, in which one $\mathrm{SH}$ group remains unimmobilized on the substrate. ${ }^{18}$ For the substrates $\mathbf{I}^{1 s t} \boldsymbol{L}$ and $\mathbf{I I}^{1 \text { st }} \boldsymbol{L}$, the conjugation of colloidal silver led to a greater decrease in frequency with increasing $1 l / 2 l$, whereas only a marginal decrease was found for both substrates $I^{2 n d} \boldsymbol{L}$ and $\mathrm{II}^{2 \text { nd }} \boldsymbol{L}$ regardless of the amount of $\mathbf{1} \boldsymbol{l} / \mathbf{2 l}$ present (Figure 3, $\mathbf{I}^{1 \mathrm{st}} L / \mathbf{I I}^{1 \mathrm{st}} L$ and $\left.\mathbf{I}^{2 \mathrm{nd}} L / \mathbf{I I}^{2 \mathrm{nd}} L\right)$. These results suggest that there were remaining $\mathrm{SH}$ groups on substrates $\mathbf{I}^{1 \mathrm{st}} \boldsymbol{L}$ and $\mathbf{I I}^{1 \mathrm{lst}} \boldsymbol{L}$, which were allowed to form loop structures, accompanied by SAM formation of 3 in the second step. In addition, the conjugation of colloidal silver after simple incubation (which is a control experiment for the second step) of the substrates $\mathbf{I}^{1 \text { st }} \boldsymbol{L}$ and $\mathbf{I I}^{1 \text { st }} \boldsymbol{L}$ in the same solvent without $\mathbf{3}$, as used in the SAM formation at the second step, decreased the frequency to some extent (Figure 3, $\mathbf{I}^{\text {inc }} \boldsymbol{L}$ and $\mathbf{I I}^{\text {inc }} \boldsymbol{L}$ ). The frequency decrease indicated that the presence of $\mathbf{3}$ in the second step is essential to the completion of loop formation.

\section{Surface properties of PEG- and polyrotaxane-immobilized} substrates $\mathrm{I}^{2 \text { nd }}$ and $\mathrm{II}^{2 \text { nd }}$

Dynamic motion of water-soluble polymers on a substrate in water has often been investigated by QCM-D measurements, which is accompanied by a larger ratio of change in energy dissipation to shift in frequency $(\Delta D / \Delta f)$ and is directly related to the softness of the polymer layer by assuming the energy loss in the viscoelastic Voigt model. ${ }^{28}$ We first discuss surface softness or rigidity of the hydrated substrates $\mathbf{I}^{\text {nd }}$ and $\mathbf{I I}^{2 \text { nd }}$ in an aqueous environment by calculating the ratio for a gold substrate in water before and after the two-step immobilization in QCM-D measurement. The results are summarized in Table 1 . The reliability of a $\Delta D / \Delta f$ value for each substrate was confirmed by the linearity of $\Delta D-\Delta f$ plots obtained from three substrates a-c with different amounts of polymers (Figure 4). For both PEG-immobilized $\mathbf{I}^{2 \text { nd }}$ and polyrotaxane-immobilized $\mathbf{I I}^{2 \mathrm{nd}}$, smaller values of $\Delta D / \Delta f$ were obtained from substrates with a loop structure $(\boldsymbol{L})$ than those with a graft structure $(\boldsymbol{G}){ }^{20}$ PEG on a substrate exhibited higher values of $\Delta D / \Delta f$ than polyrotaxane, although exactly the same PEG existed in the polyrotaxane structure in which a number of methylated $\alpha$-CD molecules are threaded and aligned along the PEG chain to restrict the mobility of PEG. Thus, on substrates in water, it seems that PEG is more flexible and gives more softness to a substrate than polyrotaxane.

The surface wettability of the substrates $\mathbf{I}^{2 \text { nd }}$ and $\mathbf{I I}^{2 \text { nd }}$ was then investigated in terms of static and dynamic contact angles. ${ }^{29} \mathrm{~A}$ relatively higher total surface tension was estimated for all substrates $\mathbf{I}^{2 \text { nd }} \boldsymbol{L} / \boldsymbol{G}$ and $\mathrm{II}^{2 \mathrm{nd}} \boldsymbol{L} / \boldsymbol{G}$ with increasing numbers of polymers $\mathbf{1} / \mathbf{g}$ and $\mathbf{2 l} / \mathbf{g}$. We found little difference in the total surface tension and dispersion/polar component among $\mathrm{I}^{2 \mathrm{nd}} \boldsymbol{L} / \boldsymbol{G}, \quad \mathbf{I I}^{2 \mathrm{nd}} \boldsymbol{L} / \boldsymbol{G}$ and III (Figure 5a). Both advancing and receding contact angles decreased with increasing numbers of polymers $1 / / g$ and $2 l / g$ on all substrates $\mathbf{I}^{2 \text { nd }} \boldsymbol{L} / \boldsymbol{G}$ and $\mathbf{I I}^{\text {2nd }} \boldsymbol{L} / \boldsymbol{G}$, which were almost indistinguishable (Figure 5b). These advancing and receding contact angles were independent of the flow rate and the time for delivery of a water droplet. Finding these similarities of contact angles on PEG-immibilized $\mathbf{I}^{2 \text { nd }}$ and polyrotaxane-immobilized $\mathbf{I I}^{2 \text { nd }}$ with different function- 


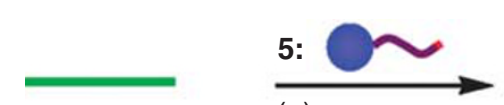

(a)

4
5:

(a)

4

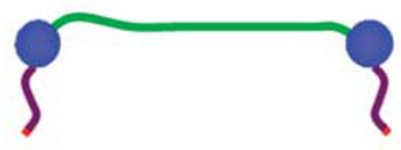

$1 /$

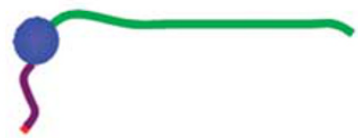

6

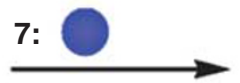

(b)

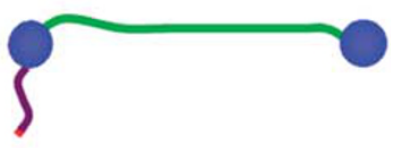

$1 g$<smiles>CC#CCNCCOCCCOCCN</smiles>

Scheme 1 Preparation of poly(ethylene glycol) (PEG) $\mathbf{1} / \mathbf{g}$ through a condensation reaction of PEG bis(2-aminoethyl) ether 4 with tyrosine $\mathbf{5} /$ tyrosine $\mathbf{5}$ and phenylalanine 7. Reagents and yields: (a) DMT-MM, MeOH (29\% for $1 /$ and $47 \%$ for 6); (b) BOP, HOBt, DIEA, dimethylformamide (DMF; $46 \%$ for 1 g).

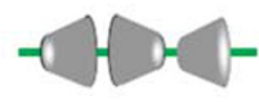

$4 \cdot \alpha-C D$

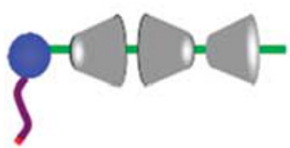

$6 \cdot \alpha-C D$
5:

(a)

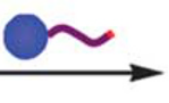

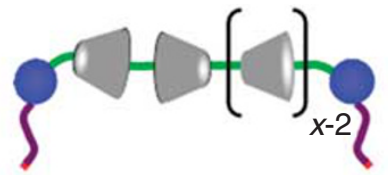

8I (average $x=19$ ) (b)

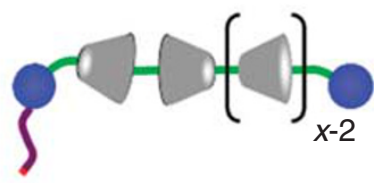

$\mathbf{8 g}$ (average $x=26$ )

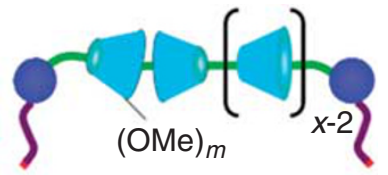

2I (average $x=19, m=11$ )

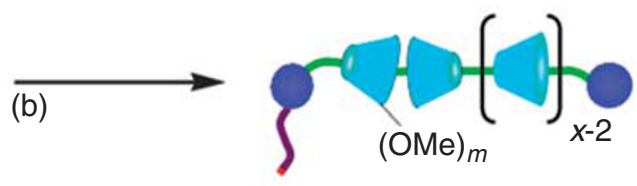

$\mathbf{2 g}$ (average $x=26, m=11$ )
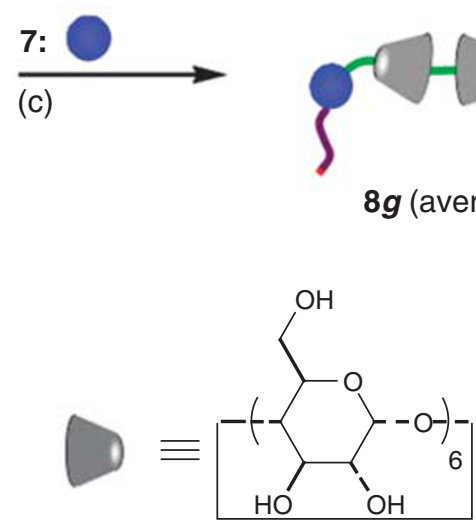

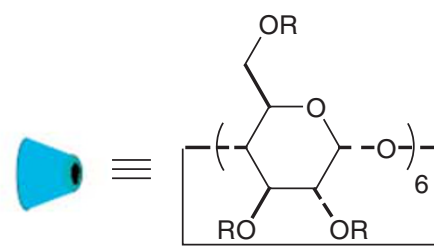

$\mathrm{R}=\mathrm{H}$ or $\mathrm{Me}$

Scheme 2 Preparation of polyrotaxane $2 / / \mathbf{g}$ through endcapping of pseudopolyrotaxane $\mathbf{4} \cdot \alpha-C D / 6 \cdot \alpha-C D$ with tyrosine $5 /$ phenylalanine 7 , followed by partial methylation of $\alpha$-CD in $\mathbf{8} / \mathbf{g}$. Reagents and yields: (a) DMT-MM, MeOH (6\% for 8 ); (b) $\mathrm{NaOH}, \mathrm{CH}_{3} \mathrm{l}$, DMSO (14\% for $2 \mathrm{I}$ and $18 \%$ for $2 \mathrm{~g}$ ); (c) $\mathrm{BOP}$, $\mathrm{HOBt}$, DIEA, dimethylformamide (DMF; $9 \%$ for $\mathbf{8 g}$ ). 


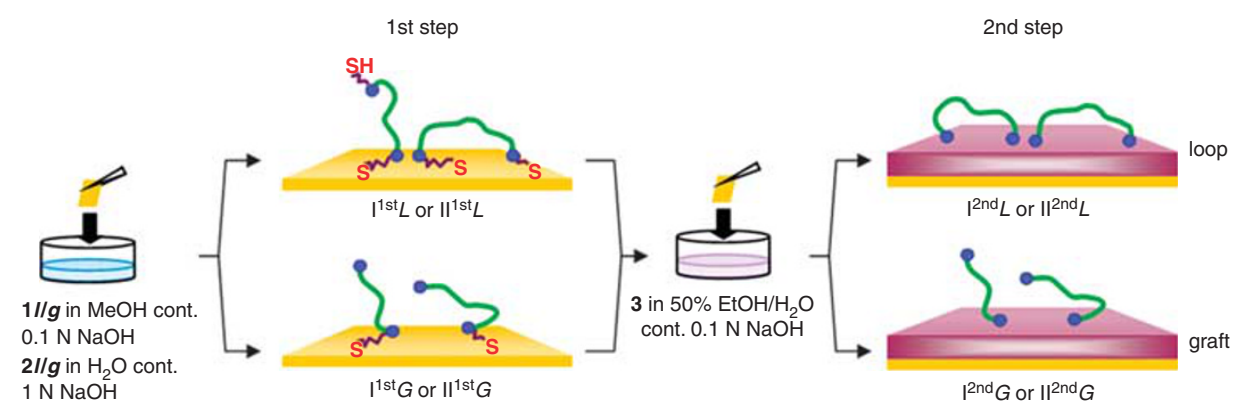

Scheme $\mathbf{3}$ Preparation of PEG- and polyrotaxane-immobilized substrates substrated $\mathbf{I}^{2 \text { nd }} \boldsymbol{L} / \mathbf{I}^{\text {2nd }} \boldsymbol{G}$ and $\mathbf{I I}^{2 \text { nd }} \boldsymbol{L} / \mathbf{I I}^{2 \text { nd }} \boldsymbol{G}$ covered with a SAM of $\mathbf{3}$ (two-step protocol),

Table 1 Immobilization conditions (concentration and immersion time), results from the first step (change in mass $\left(\Delta m_{1-0}\right)^{\mathrm{a}}$ of a gold

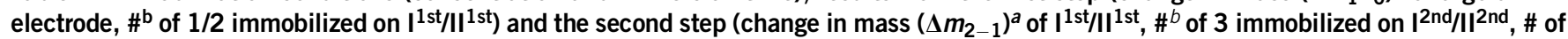
$\mathrm{OH}$ and $\mathrm{OMe}$ groups on $\mathrm{I}^{2 \mathrm{nd}}$, II ${ }^{2 \mathrm{nd}}$, and III), measured by QCM $(n=3)$, and the $\Delta D / \Delta f$ values, measured by QCM-D $(n=3)$

\begin{tabular}{|c|c|c|c|c|c|c|c|c|c|}
\hline \multirow[b]{2}{*}{ Substrate } & \multicolumn{4}{|c|}{$1^{s t}$} & \multicolumn{4}{|c|}{$2^{\text {nd }}$} & \multirow[b]{2}{*}{$\begin{array}{c}\Delta D / \Delta f \\
\left(\times 10^{-9}\right)\end{array}$} \\
\hline & $\begin{array}{l}{[1-2]} \\
\left(\times 10^{-6}{ }_{M}\right)\end{array}$ & Immersion time & $\begin{array}{c}\Delta m_{1-0} \\
\left(\times 10 \mathrm{ngcm}^{-2}\right)\end{array}$ & $\begin{array}{l}\text { \# of } 1-2 \\
\left(/ 100 \mathrm{~nm}^{2}\right)\end{array}$ & $\begin{array}{c}\Delta m_{2-1} \\
\left(\times 10^{2} \mathrm{ngcm}^{-2}\right)\end{array}$ & $\begin{array}{c}\# \text { of } \mathbf{3}\left(\times 10^{2}\right. \\
\left.\quad 1100 \mathrm{~nm}^{2}\right)\end{array}$ & $\begin{array}{l}\#_{O H}\left(\times 10^{2}\right. \\
\left./ 100 \mathrm{~nm}^{2}\right)\end{array}$ & $\begin{array}{c}\#_{O M e}\left(\times 10^{2}\right. \\
\left./ 100 \mathrm{~nm}^{2}\right)\end{array}$ & \\
\hline \multicolumn{10}{|l|}{ IL } \\
\hline a & $1.0 \times 10^{-10}$ & $10 \mathrm{~s}$ & 0.8 & 1.1 & 1.8 & 3.1 & 3.1 & - & 8.6 \\
\hline b & $1.0 \times 10^{-10}$ & $30 \mathrm{~s}$ & 1.6 & 2.2 & 1.8 & 3.1 & 3.1 & - & 8.6 \\
\hline a & $1.0 \times 10^{-10}$ & $10 \mathrm{~s}$ & 0.8 & 1.2 & 1.8 & 3.1 & 3.1 & - & 11 \\
\hline b & $1.0 \times 10^{-10}$ & $30 \mathrm{~s}$ & 1.4 & 2.1 & 1.8 & 3.1 & 3.1 & - & 11 \\
\hline c & $1.0 \times 10^{-9}$ & $60 s$ & 1.8 & 2.7 & 1.8 & 3.1 & 3.1 & - & 11 \\
\hline \multicolumn{10}{|l|}{ IIL } \\
\hline a & 0.10 & $10 \mathrm{~min}$ & 5.1 & 1.2 & 1.8 & 3.1 & 4.7 & 2.5 & 2.3 \\
\hline b & 0.10 & $20 \mathrm{~min}$ & 9.1 & 2.1 & 1.8 & 3.1 & 5.9 & 4.4 & 2.5 \\
\hline c & 1.0 & $30 \mathrm{~min}$ & 19 & 3.4 & 1.8 & 3.1 & 9.3 & 9.9 & 5.3 \\
\hline III & - & - & - & - & 1.8 & 3.1 & 3.1 & - & 0.4 \\
\hline
\end{tabular}

Abbreviations: QCM, quartz crystal microbalance; QCM-D, QCM with dissipation monitoring.

${ }^{a} \Delta m_{1-0}$ and $\Delta m_{2-1}$ were calculated according to the Sauerbrey equation to estimate the numbers of immobilized $\mathbf{1 / 2}$ and $\mathbf{3}$, respectively.

${ }^{b}$ The numbers of immobilized $1 \mathrm{l}, \mathbf{1} \mathrm{g}, \mathbf{2 l}, \mathbf{2} \mathrm{g}$ and $\mathbf{3}$ were calculated based on molecular weights of ca $4.2 \times 10^{3}$ for $1 \mathrm{l}$, ca $3.9 \times 10^{3}$ for $1 \mathrm{~g}$, ca $2.6 \times 10^{4}$ for $\mathbf{2 l}$, ca $3.4 \times 10^{4}$ for $2 \mathrm{~g}$ and 350 for $\mathbf{3}$.

ality (a number of methoxy groups exist only on $\mathbf{I I}^{2 \text { nd; }}$ Table 1) was unexpected. The immobilization modes, $\boldsymbol{L}$ and $\boldsymbol{G}$, did not influence contact angles.

We investigated the adsorption of two proteins, HSA and HPF, onto substrates $\mathbf{I}^{\text {2nd }}$ and $\mathbf{I I}^{2 \text { nd }}$ using SPR spectroscopy. These two major proteins are commonly used to study platelet adhesion and the subsequent events leading to thrombosis. ${ }^{1,30}$ HSA was less well adsorbed than expected on all substrates $\mathbf{I}^{2 \mathrm{nd}} \boldsymbol{L} / \boldsymbol{G}, \mathbf{I I}^{2 \mathrm{nd}} \boldsymbol{L} / \boldsymbol{G}$ and III $\left(0.03-0.06 \mu \mathrm{g} \mathrm{cm}^{-2}\right)$ during a solution flow $\left(4.5 \mathrm{mg} \mathrm{ml}^{-1}, 15 \mu \mathrm{min}^{-1}\right.$, $5 \mathrm{~min}$; Figure 6). Additional interesting results were obtained from a solution flow of HPF $\left(0.3 \mathrm{mg} \mathrm{ml}^{-1}, 15 \mu \mathrm{min}^{-1}, 5 \mathrm{~min}\right)$ on polyrotaxane-immobilized substrates $\mathbf{I I}^{2 \mathrm{nd}} \boldsymbol{L}$ and $\mathbf{I I}^{2 \mathrm{nd}} \boldsymbol{G}$. HPF adsorption on the loop surface $(\boldsymbol{L})\left(\sim 0.2 \mu \mathrm{g} \mathrm{cm}^{-2}\right)$ was less than that on the graft surface (G) $\left(0.2 \sim 0.3 \mu \mathrm{g} \mathrm{cm}^{-2}\right)$ and comparable to III $\left(0.2 \mu \mathrm{g} \mathrm{cm}^{-2}\right.$; Figure 7$)$. On PEG-immobilized substrates $\mathbf{I}^{\text {2nd }} \boldsymbol{L}$ and $\mathbf{I}^{2 \text { nd }} \boldsymbol{G}$, the difference in adsorption seemed to be negligible $\left(0.3 \mu \mathrm{g} \mathrm{cm}^{-2}\right)$. Under a more severe condition $\left(0.3 \mathrm{mg} \mathrm{ml}^{-1}, 1 \mu \mathrm{min}^{-1}, 75 \mathrm{~min}\right)$, all surfaces of
$\mathbf{I}^{2 \text { nd }} \boldsymbol{L} / \boldsymbol{G} \mathbf{b}, \mathbf{I I}^{2 \text { nd }} \boldsymbol{L} / \mathbf{G} \mathbf{b}$ and III showed a similar trend to that found in Figure 7 with a small increase in protein adsorption (Supplementary Figure S3 in the Supplementary Information). These protein adsorptions appear to be independent of the amounts of polymers $(\mathbf{a}-\mathbf{c}$, Figure 6 and Figure 7).

If we consider the results regarding surface wettability (Figure 5), which is similar for all substrates, it is difficult to explain the difference or similarity in HPF adsorption on polyrotaxane-immobilized substrates $\mathbf{I I}^{2 \text { nd }} \boldsymbol{L}$ and $\mathbf{I I}^{2 \text { nd }} \boldsymbol{G}$ or PEG-immobilized $\mathbf{I}^{2 \text { nd }} \boldsymbol{L}$ and $\mathbf{I}^{\text {2nd }} \boldsymbol{G}$. Instead, we tried to explain the difference or similarity as being due to the softness or rigidity of the substrates (Figure 4). One can find a correlation that less HPF was adsorbed on less-flexible substrates.

\section{CONCLUSION}

We designed and prepared four types of SAMs, $\mathbf{I}^{2 \mathrm{nd}} \boldsymbol{L} / \boldsymbol{G}$ and $\mathbf{I I}^{2 \mathrm{nd}} \boldsymbol{L} / \boldsymbol{G}$, on which water-soluble polymers, PEG $1 / / g$ and polyrotaxane $2 l / g$, were immobilized, respectively, in loop $(\boldsymbol{L})$ or graft mode $(\boldsymbol{G})$, and 


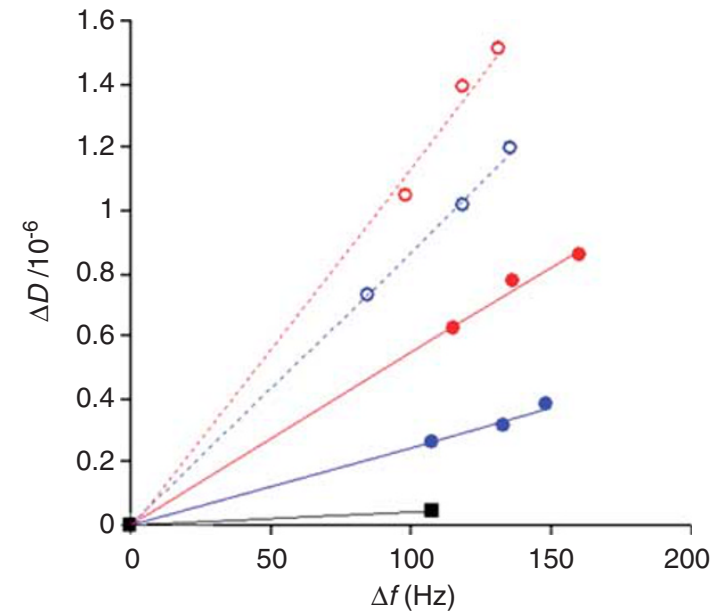

Figure 4 Plots of change in dissipation factor $(\Delta D)$ against shift in frequency $(\Delta f)$ (red circle for $\mathbf{I}^{\text {nd }} \boldsymbol{L}$, open red circle for $\mathbf{I}^{2 \text { nd }} \boldsymbol{G}$, blue circle for $\mathbf{I I}^{2 \text { nd }} \boldsymbol{L}$, open blue circle for II $^{2 \text { nd }} \boldsymbol{G}$, and black square for III) measured by quartz crystal microbalance with dissipation monitoring $(n=3)$.
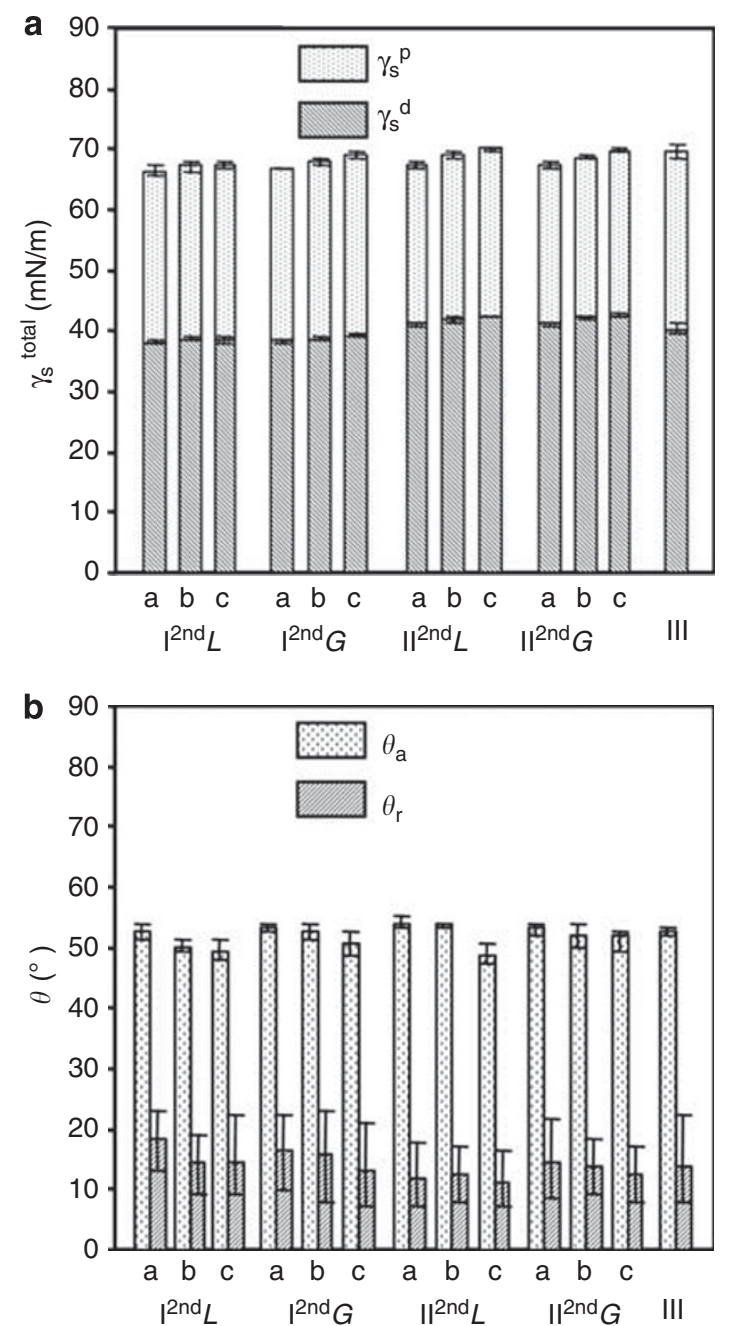

Figure 5 (a) Total surface tensions $\left(\gamma_{s}^{\text {total }}\right)$, the sums of dispersive $\left(\gamma_{s}{ }^{d}\right)$ and polar $\left(\gamma_{\mathrm{s}}{ }^{\mathrm{p}}\right)$ surface tensions $(n=3)$ and $(\mathbf{b})$ advancing $\left(\theta_{\mathrm{a}}\right)$ and receding $\left(\theta_{\mathrm{r}}\right)$ contact angles $(n=3)$.

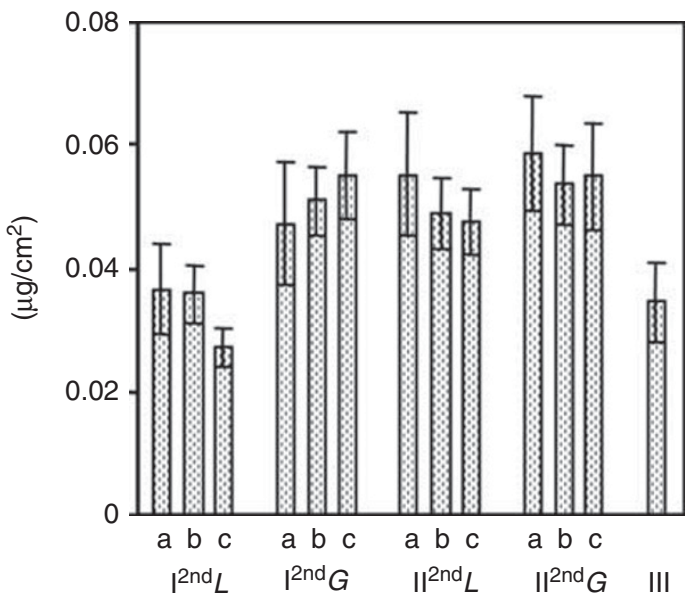

Figure 6 Amounts of human serum albumin adsorbed on substrates $\mathbf{I}^{\text {2nd }}$, $\mathrm{II}^{\text {2nd }}$ and $\mathbf{I I I}$, measured by surface plasmon resonance $(n=3)$.

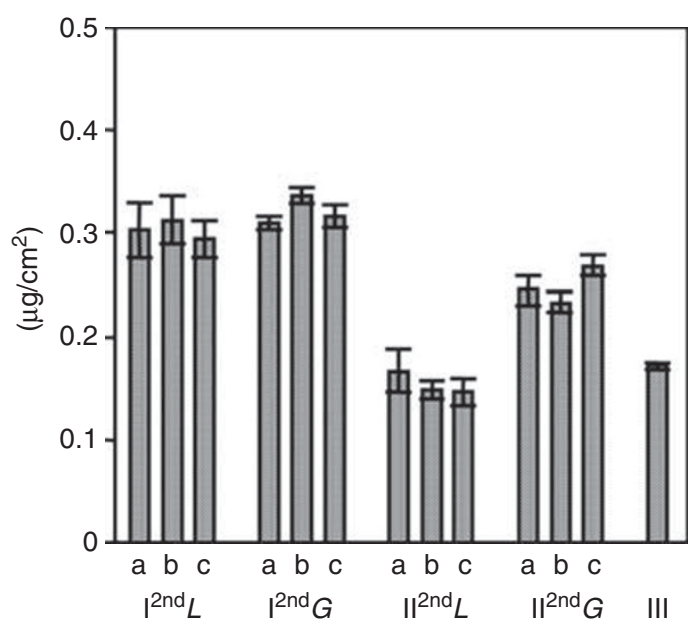

Figure 7 Amounts of human plasma fibrinogen (HPF) adsorbed on substrates $\mathbf{I}^{2 \text { nd }}, \mathbf{I I}^{2 \text { nd }}$ and $\mathbf{I I I}$, measured by surface plasmon resonance $\left(0.3 \mathrm{mg} \mathrm{ml}^{-1}, 15 \mu \mathrm{min}^{-1}, 5 \mathrm{~min}, n=3\right)$. HPF was adsorbed slightly above the present level under a more severe condition $\left(0.3 \mathrm{mg} \mathrm{ml}^{-1}, 1 \mu \mathrm{lmin}{ }^{-1}\right.$, $75 \mathrm{~min}, n=3$, Supplementary Figure S3).

examined protein adsorption on these SAMs. The loop and graft formations on SAMs were successfully controlled by the two-step protocol and monitored by QCM. QCM-D measurement demonstrated surface softness or rigidity of the hydrated polymers on SAMs in water, showing the smallest value of $\Delta D / \Delta f$ for the polyrotaxane loop $\left(\mathbf{I I}^{2 n d} \boldsymbol{L}\right)$ and the highest for the PEG graft $\left(\mathbf{I}^{2 \text { nd }} \boldsymbol{G}\right)$. In spite of these differences in polymer and/or structure on SAMs, the surface wettability of the SAMs is similar in terms of static and dynamic contact angles. From plasma protein adsorption tests, we found that the polyrotaxane loop $\left(\mathbf{I I}^{2 \mathrm{nd}} \boldsymbol{L}\right)$ exhibited a much better non-fouling property for the adsorption of HPF than the PEG loop and graft ( $\mathbf{I}^{2 \text { nd }} \boldsymbol{L}$ and $\mathbf{I}^{2 \text { nd }} \boldsymbol{G}$ ), and is comparable to the reference SAM III, which is already known to be effective in eliminating protein adsorption. Although the details of the mechanism are still unclear, the loop formation of a polyrotaxane can be responsible for reducing fibrinogen adsorption related to platelet adhesion and activation, and may provide a possibility for designing biomaterials based on polyrotaxane loops. 


\section{ACKNOWLEDGEMENTS}

We thank Associate Professor Akio Ohta (Kanazawa University) and Dr Issey Osaka (JAIST) for their help in the characterization (EI, FAB, ESI and MALDITOF mass spectrometry) of the new compounds. We also thank Professor Kazuhiko Ishihara and Dr Yuuki Inoue (The University of Tokyo) for supplying the Au-evaporated silicon wafers.

1 Gorbet, M. B. \& Sefton, M. V. Biomaterial-associated thrombosis: roles of coagulation factors, complement, platelets and leukocytes. Biomaterials 25, 5681-5703 (2004).

2 Shoichet, M. S. Polymer scaffolds for biomaterials applications. Macromolecules 43, 581-591 (2010).

3 Chen, H. C., Yuan, L., Song, W., Wu, Z. \& Li, D. Biocompatible polymer materials: role of protein surface interactions. Prog. Polym. Sci. 33, 1059-1087 (2008).

4 Jeon, S. I., Lee, J. H., Andrade, J. D. \& De Gennes, P. G. Protein-surface interactions in the presence of polyethylene oxide: I. Simplified theory. J. Colloid Interf. Sci. 142, 149-158 (1991).

5 Jeon, S. I. \& Andrade, J. D. Protein-surface interactions in the presence of polyethylene oxide: II. Effect of protein size. J. Colloid Interf. Sci. 142, 159-166 (1991).

6 Currie, E. P. K., Norde, W. \& Cohen Stuart, M. A. Tethered polymer chains: surface chemistry and their impact on colloidal and surface properties. Adv. Colloid Interf. Sci. 100-102, 205-265 (2003)

7 Unsworth, L. D., Sheardown, H. \& Brash, J. L. Protein resistance of surfaces prepared by sorption of end-thiolated poly(ethylene glycol) to gold: effect of surface chain density. Langmuir 21, 1036-1041 (2005).

8 Zheng, J., Li, L., Tsao, H.- K., Sheng, Y.- J., Chen, S. \& Jiang, S. Strong repulsive forces between protein and oligo(ethylene glycol) self-assembled monolayers: a molecular simulation study. Biophys. J. 89, 158-166 (2005).

9 Ishihara, K., Nomura, H., Mihara, T., Kurita, K., Iwasaki, Y. \& Nakabayashi, N. Why do phospholipid polymers reduce protein adsorption? J. Biomed. Mater. Res. 39, 323-330 (1998).

10 Yamasaki, A., Imamura, Y., Kurita, K., Iwasaki, Y., Nakabayashi, N. \& Ishihara, K. Surface mobility of polymers having phosphorylcholine groups connected with various bridging units and their protein adsorption-resistance properties. Colloids Surf. B 28, 53-62 (2003)

11 Kitano, H., Imai, M., Mori, T., Gemmei-Ide, M., Yokoyama, Y. \& Ishihara, K. Structure of water in the vicinity of phospholipid analogue copolymers as studied by vibrational spectroscopy. Langmuir 19, 10260-10266 (2003).

12 Feng, W., Zhu, S., Ishihara, K. \& Brash, J. L. Adsorption of fibrinogen and lysozyme on silicon grafted with poly(2-methacryloyloxyethyl phosphorylcholine) via surfaceinitiated atom transfer radical polymerization. Langmuir 21, 5980-5987 (2005).

13 He, Y., Hower, J., Chen, S., Bernards, M. T., Chang, Y. \& Jiang, S. Molecula simulation studies of protein interactions with zwitterionic phosphorylcholine self-assembled monolayers in the presence of water. Langmuir 24, 10358-10364 (2008).

14 Prime, K. L. \& Whitesides, G. M. Adsorption of proteins onto surfaces containing endattached oligo(ethylene oxide): a model system using self-assembled monolayers. J. Am. Chem. Soc. 115, 10714-10721 (1993)

15 Sigal, G. B., Mrksich, M. \& Whitesides, G. M. Effect of surface wetability on the adsorption of proteins and detergents. J. Am. Chem. Soc. 120, 3464-3473 (1998).

16 Benesch, J., Svedhem, S., Svensson, S. C. T., Valiokas, R., Liedberg, B. \& Tengvall, P. Protein adsorption to oligo(ethyelene glycol) self-assembled monolayers: experiments with fibrinogen, heparinized plasma, and serum. J. Biomater. Sci. Polym. Edn. 12, 581-597 (2001).

17 Lu, H. B., Campbell, C. T. \& Castner, D. G. Attachment of functional poly(ethylene glycol) films to gold surfaces. Langmuir 16, 1711-1718 (2000).

18 Yang, D. H., Katoono, R., Yamaguchi, J., Muira, Y. \& Yui, N. Immobilization of polyrotaxane on a solid substrate as the design of dynamic surface. Polym. J. 41, 952-953 (2009).

19 Kim, Y.- H. \& Gorman, C. B. Standing up versus looping over: controlling the geometry of self-assembled monolayers of $\alpha, \omega$-diynes on gold. Langmuir 27, 6069-6075 (2011).

20 Patton, D., Knoll, W. \& Advincula, R. C. Polymer loops vs brushes on surfaces: adsorption, kinetics, and viscoelastic behavior of $\alpha, \omega$-thiol telechelics on gold. Macromol. Chem. Phys. 212, 485-497 (2011).

21 Ulman, A. Formation and structure of self-assembled monolayers. Chem. Rev. 96, 1533-1554 (1996).

22 Wang, W., Efrima, S. \& Regev, O. Directing oleate stabilized nanosized silver colloids into organic phases. Langmuir 14, 602-610 (1998).

23 Buttry, D. A. \& Ward, M. D. Measurement of interfacial processes at electrode surfaces with the electrochemical quartz crystal microbalance. Chem. Rev. 92, 1355-1379 (1992).

24 Rodahl, M., Höök, F., Krozer, A., Brzezinski, P. \& Kasemo, B. Quartz crystal microbalance setup for frequency and $Q$-factor measurements in gaseous and liquid environments. Rev. Sci. Instrum. 66, 3924-3930 (1995).

25 Owens, D. K. \& Wendt, R. C. Estimation of the surface free energy of polymers. J. Appl. Polym. Sci. 13, 1741-1747 (1969)

26 Stavenger, R. A. \& Schreiber, S. L. Asymmetric catalysis in diversity-oriented organic synthesis: enantioselective synthesis of 4320 encoded and spatially segregated dihydropyrancarboxamides. Angew. Chem. Int. Ed. 40, 3417-3421 (2001).

27 Anyaogu, K. C., Fedorov, A. V. \& Neckers, D. C. Synthesis characterization, and antifouling potential of functionalized copper nanoparticles. Langmuir 24, 43404346 (2008).

28 Voinova, M. V., Rodahl, M., Jonson, M. \& Kasemo, B. Viscoelastic acoustic response of layered polymer films at fluid-solid interfaces: continuum mechanics approach. Physica Scripta 59, 391-396 (1999).

29 Garbassi, F., Morra, M. \& Occhiello, E. in Polymer Surfaces: From Physics to Technology, Ch. 4. (John Wiley \& Sons: New York, 1996.

30 Horbett, T. A. \& Brash, J. L. Proteins at Interfaces II: Fundamentals and Applications. (American Chemical Society: Washington DC, 1995).

Supplementary Information accompanies the paper on Polymer Journal website (http://www.nature.com/pj) 